provision of information and in providing support, has often been underestimated. This survey shows that, when available, their support is usually welcomed by both clients and carers and unmet needs are less.

In conclusion, deficiencies have been found in monitoring adults with severe physical disability whose sole regular contacts are health professionals. District health authorities and family health services authorities will need to consider how best to improve the assessment of needs of patients as they plan to implement care in the community.

1 Secretaries of State for Health. Caring for people. London: HMSO, 1989 2 Secretaries of State for Health. NHS and community care act. London: HMSO, 1990.

3 Cantrell E, Dawson J, Glastonbury G. Prisoners of handicap. London: RADAR, 1985.

4 Martin J, Meltzer $\mathrm{H}$, Elliot $\mathrm{D}$. The prevalence of disability among adults. OPCS surveys of disability in Great Britain. Report 1. London: HMSO, 1988. 5 Culyer AJ. Need and the National Health Service. London: Martin Robertson 1976.
6 Williams A. Need-an economic exegesis. In: Culyer AJ, Wright KG, eds. Economic aspects of the health service. London: Martin Robertson, 1978.

Buchan H, Gray JAM, Hill A, Coulter A. Needs assessment made simple. Health Services foumal 1990;100:240-1.

8 Bradshaw J. A taxonomy of social need. In: McLachlan G, ed. Problems and progress in medical care: essays on current research. London: Oxford University Press, 1972.

9 Edwards FC, Warren MD. Health services for adults with physical disabilities. London: Royal College of Physicians, 1990.

10 Beardshaw V. Last on the list. London: King's Fund, 1988. (Research report 3.)

11 Patrick DL, Peach H, Gregg I. Disablement and care: a comparison of patient views and general practitioner knowledge. I R Coll Gen Pract 1982;32: 429-34.

12 Thomas A, Bax M, Coombs K, Goldson E, Smyth D, Whitmore K. The health and social needs of physically handicapped young adults: are they being me by the statutory services? Dev Med Child Neurol 1985;4(suppl):50.

13 Blaxter M. Principles and practice in rehabilitation. In: Report of the royal commission on the National Health Service. London: HMSO, 1979. (Cmnd 7615.)

14 Brechin A, Liddiard P. Look at it this way: new perspectives in rehabilitation. London: Hodder and Stoughton in association with Open University Press, 1981.

15 Robinson T. In worlds apart. London: Bedford Square Press, 1978

6 Campbell MJ, Enderby P. The management of motor neurone disease. f Neurol Sci 1984;64:65-71.

(Accepted 30 October 1992)

\title{
Long term follow up of severely ill patients who underwent urgent cardiac transplantation
}

\author{
D Mulcahy, M Fitzgerald, C Wright, J Sparrow, J Pepper, M Yacoub, K M Fox
}

\section{Abstract}

Objective-To assess long term survival $(>5$ years) and quality of life in severely ill patients referred for urgent cardiac transplantation.

Setting-Tertiary referral centres: before transplantation at the National Heart Hospital (late 1984 to end 1986); after transplantation at Harefield Hospital.

Subjects-Eighteen patients $(15$ men; three women) who had required intensive support in hospital before cardiac transplantation and were alive at short term follow up.

Interventions-Intravenous infusions of cardiac drugs (mean 2.2 infusions), intravenous diuretics (17 patients), and many other drugs before transplantation. Intra-aortic balloon counterpulsation (four patients), temporary pacing (two), and rescusitation from cardiac arrest (three). Patients had specialised nursing care on a medical intensive care unit in almost every case.

Main outcome measures-Long term survival in patients after urgent cardiac transplantation and perceived quality of life.

Results-Of 18 patients who were alive at short term follow up (mean (range) 19.4 (10-33) months), 14 were still alive in 1992 (69 (61-83) months). Ten still worked full time, and 11 reported no restrictions in their daily activities. Three of four patients who died in the intervening period survived $>5$ years after transplantation. Overall, 17 of 18 patients survived at least 5 years.

Conclusions-In severely ill patients who undergo urgent cardiac transplantation and survive in the short term, long term (5-7 year) survival and quality of life seem good.

\section{Introduction}

Cardiac transplantation has now become an accepted therapeutic option for many patients with terminal cardiac failure. A total of 3054 heart transplantations were reported to the registry of the International Society of Heart and Lung Transplantation in 1990, and overall actuarial survival rates at 1 and 5 years have been reported at $90 \%$ and $68 \%$ respectively. Of more than 16000 heart transplantations performed since the first human to human operation in 1967, over 1700 patients survived more than 5 years after surgery. ${ }^{\prime}$ The success of this fairly new treatment has led to increasing numbers of terminally ill patients being referred for consideration of transplantation, many of these being referred as a final option, when intensive support in hospital is required and when secondary end organ damage has often developed. Organ donation has not kept pace with the demands for transplantation, ${ }^{2}$ and a considerable minority of patients die having been accepted for, and while waiting for, transplantation. With the chronic shortage of donor organs a dilemma faces the physician who is referred a patient with terminal heart failure with or without secondary end organ damage who requires intensive support in hospital. Should treatment be withheld and the available donor organs donated to "more suitable" patients or should such ill patients be actively treated while waiting for a suitable donor organ, thus leading to a potential increase in the number of stable patients likely to deteriorate while awaiting "elective" transplantation?

In early 1988 we reported in this journal the short term outcome in 33 patients referred to the National Heart Hospital between late 1984 and the end of 1986 and who were accepted for urgent cardiac transplantation. ${ }^{3}$ All required intensive treatment in hospital with intravenous infusions of cardiac drugs, in addition to various combinations of intra-aortic balloon counterpulsation, peritoneal dialysis, ventilation, and temporary pacing to sustain life, and none had any prospect of discharge from hospital without a transplant. The seven patients who did not receive a transplant died in hospital, but 18 of 20 patients who survived to hospital discharge after transplantation were alive at short term follow up (mean (range) $19 \cdot 4$ (10-33) months). In this report we assess the long term (5-7 year) survival, exercise capacity, perceived quality of life, and employment in these 18 patients.

\section{Patients and methods}

Follow up data for the 18 patients who were alive when the 1988 report was assembled have been

Dr Mulcahy. 
obtained from the most recent inpatient assessment at Harefield Hospital (1991-2), where all but one patient are reviewed in depth each year or more often if problems arise. Details of exercise capacity, coronary anatomy, left ventricular ejection fraction, and medications taken were recorded from the notes, in addition to renal functional state, blood pressure, and associated medical problems.

\section{QUALITY OF LIFE AND EMPLOYMENT}

Survivors were contacted by telephone over two days in January 1992. Patients underwent a simple assessment of quality of life with regard to their perceived restrictions and their employment; their exercise capacity had been assessed with exercise testing during their most recent annual follow up visit. Patients were asked (by DM or CW) whether they felt unrestricted, mildly restricted, moderately restricted, or severely restricted in their normal daily activities. Their employment was recorded as full time (a housewife was considered as being employed full time), part time, retired, or unemployed. Reasons for lack of employment were noted.

DETAILS OF PATIENTS BEFORE AND AT TRANSPLANTATION

Of the 18 patients ( 15 men, three women) surviving short term follow up, all had required at least one intravenous infusion of an inotropic agent or vasodilator (mean (range) $2 \cdot 2(1-5)$ infusions) before transplantation in addition to intravenous diuretics in 17 cases. Other drugs were used as required (anticoagulant agents (usually intravenous heparin), vasodilators, digoxin, diamorphine, plasma expanders). Ten patients had non-sustained ventricular tachycardia or received anti-arrhythmic treatment, or both. Seven patients received antibiotics for sepsis. Three patients had clinically suspected or proved pulmonary emboli.

The patients' clinical state was usually of hypotension and sinus tachycardia, with a third heart sound almost invariably heard. In 13 of 18 cases clinical examination confirmed evidence of biventricular failure.

Four patients required intra-aortic balloon counterpulsation. This was planned but abandoned in a fifth patient when a suitable heart became available. Indication for such an intervention was usually a combination of developing oliguria with decreasing peripheral temperature. Two patients required cardiopulmonary resuscitation at some point in the hospital admission before transplantation, a further patient requiring rescusitation after an angiographic procedure. Two patients required temporary pacing. In those 11 patients who had been well enough to undergo radionuclide ventriculography, the mean left ventricular ejection fraction was $11 \cdot 7 \%$ (range $6-20 \%$ ). Otherwise cross sectional echocardiography confirmed globally poor left ventricular function. Almost all patients were restricted to bed in the medical intensive care unit during the index admission while waiting a suitable donor organ.

Four patients had considerable disturbance in renal and hepatic function. Normal or near normal renal function was classified as previously described as a plasma urea concentration of $<14 \mathrm{mmol} / \mathrm{l}$ and plasma creatinine concentration of $<160 \mu \mathrm{mol} / \mathrm{l}$; moderate renal dysfunction as that when concentrations were higher than those quoted above; and severe renal dysfunction as that when renal dialysis was instituted. Hepatic dysfunction was defined as greater than twice normal activity of at least two hepatic enzymes. ${ }^{3}$

UNDERL YING DISEASE AND TYPE OF TRANSPLANTATION

All patients had end stage cardiac failure not amenable to medical treatment or persistent ischaemia associated with poor left ventricular function. The indication for transplantation for the 18 patients surviving in the short term was ischaemic heart muscle disease in 11 patients, dilated cardiomyopathy in six, including two with puerperal cardiomyopathy, and transient myocardial ischaemia with poor left ventricular function in one. No patient had any coexistent illness apart from pulmonary emboli in three cases and secondary end organ dysfunction in four. Sixteen patients received an orthotopic heart transplant, two receiving a heterotopic (piggyback) transplant.

\section{Results}

Of the 18 patients who were alive at the time of the 1988 report, ${ }^{3} 14$ patients (11 men and three women, mean age 48 years) were alive and well in 1992 at a mean (range) of 69 (61-83) months after transplantation. Of the intervening deaths recorded, one patient died suddenly 42 months after transplantation (heterotopic heart transplant and previous coronary artery vein grafts to the recipient heart) and another died of acute myocardial infarction at 65 months, having undergone percutaneous transluminal coronary angioplasty to a lesion of the left anterior descending coronary artery of the transplanted heart the previous year. A third patient died suddenly after rupture of an aortic aneurysm 63 months after orthotopic transplantation, having been previously well and working full time. A fourth died 61 months after orthotopic transplantation of carcinoma of the lung. Therefore, three of the four documented deaths over the intervening period occurred in those who had survived for at least 5 years after surgery.

RENAL AND HEPATIC STATE BEFORE TRANSPLANTATION AND OUTCOME

Figure 1 shows the relation between renal state before transplantation in the original cohort of 33 patients described in the 1988 report and long term (5-7 year) survival. Eleven of 14 patients with normal or nearly normal renal function and three of five with moderate renal dysfunction (mean urea concentration $27.2 \mathrm{mmol} / \mathrm{l}$; mean creatinine concentration $210 \mu \mathrm{mol} /$ 1) who were discharged from hospital after transplantation remained alive in the long term.



FIG 1-Cumulative survival (to fanuary 1992) of 20 patients after discharge from hospital having undergone cardiac transplantation between Fanuary 1985 and December 1986. Follow up. Death

Of the original cohort of 33 , six of 14 patients with moderate hepatic dysfunction were discharged from hospital after transplantation, and four remained alive and well at long term follow up (three also had moderate renal dysfunction). Fourteen of 19 patients with normal or mildly abnormal hepatic function were discharged from hospital after successful transplantation, 10 being alive at long term follow up.

Of the six patients who underwent intra-aortic balloon counterpulsation and were discharged from 
hospital after transplantation two are alive, the four others having died at 10 weeks, 31,42 , and 61 months.

CLINICAL STATE AND QUALITY OF LIFE

Table I shows the excellent preservation of left ventricular ejection fraction in the long term in the surviving patients: 11 of 14 patients performed more than 9 minutes of exercise with the standard Bruce protocol at the time of their most recent annual review (1991-2). Despite the routine use of cyclosporin only two patients had a plasma creatinine concentration above $175 \mu \mathrm{mol} / \mathrm{l}$. Four patients had evidence of coronary artery disease on routine annual coronary arteriographic assessment but only two had significant disease. Seven had hypertension, with nine having hypercholesterolaemia. Table II lists quality of life score, employment, and number of family dependants. To the question "How would you describe the restrictions to your daily activities: unrestricted, mildly restricted, moderately restricted, or severely restricted?" 11 of 14 reported no restrictions, only one reporting more than mild restrictions. Ten were working full time when interviewed, only two being unemployed (one by choice since surgery; the second for medical reasons).

OVERALL SURVIVAL

Of the 26 patients who received a suitable donor organ, 17 of the 20 patients who survived to hospital

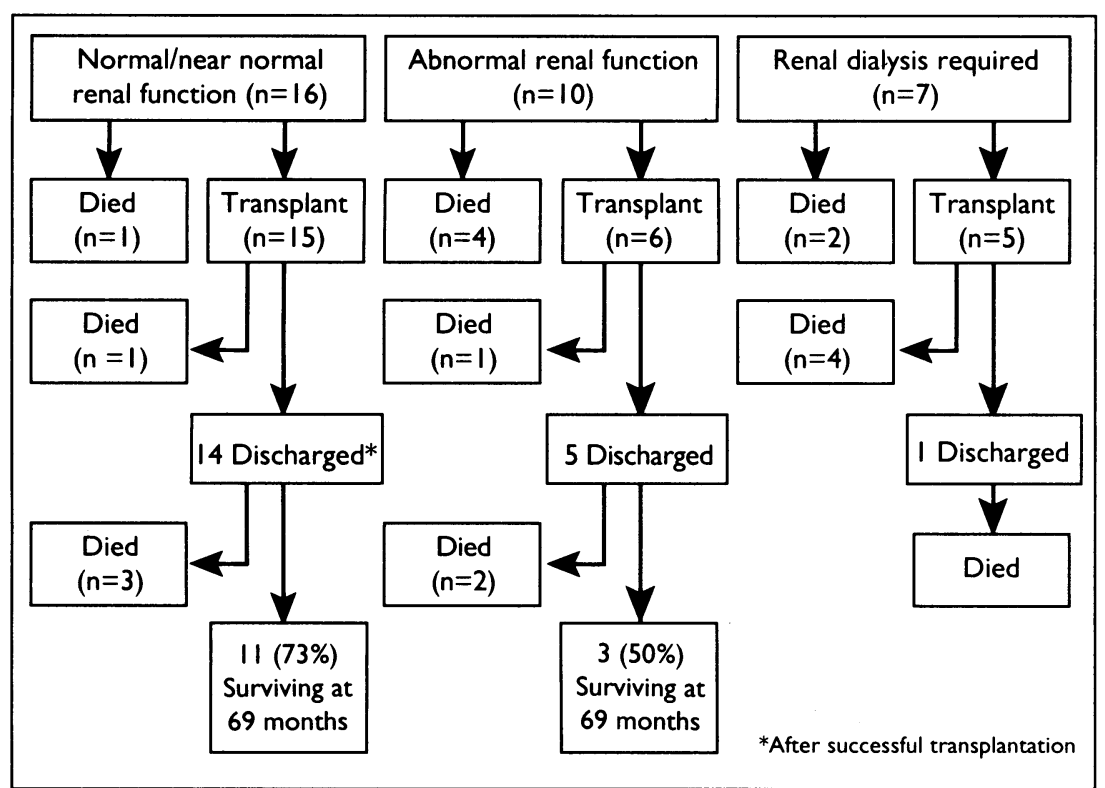

FIG 2-Long term results of cardiac transplantation when related to state of renal function before surgery in original cohort of 33 patients accepted for urgent cardiac transplantation. Normal or near normal renal function=plasma urea $<14 \mathrm{mmoll}$, plasma creatinine $<160 \mu \mathrm{molh}$; moderate renal dysfunction=urea $>14$ mmoll, creatinine $>160 \mu \mathrm{mol} / \mathrm{l}$ but not requiring renal dialysis

TABLE I-Details of patients who underwent cardiac transplantation. All information accumulated from most recent annual inpatient assessment at transplant centre that performed follow up

\begin{tabular}{|c|c|c|c|c|c|c|}
\hline $\begin{array}{l}\text { Case } \\
\text { No }\end{array}$ & $\begin{array}{l}\text { Coronary } \\
\text { anatomy }\end{array}$ & $\begin{array}{c}\text { Left ventricular } \\
\text { ejection } \\
\text { fraction } \\
(\%)\end{array}$ & $\begin{array}{l}\text { Maximum exercise } \\
\text { time with Bruce } \\
\text { protocol } \\
\text { (min) }\end{array}$ & Hypertension & $\begin{array}{c}\text { Hyper- } \\
\text { cholesterolaemia }\end{array}$ & $\begin{array}{c}\text { Plasma } \\
\text { creatinine } \\
\text { concentration } \\
(\mu \mathrm{mol} / /)\end{array}$ \\
\hline 1 & Normal & 83 & $15 \cdot 2$ & Yes & Yes & 173 \\
\hline 2 & Normal & 59 & $9 \cdot 2$ & No & No & 129 \\
\hline $3^{\star}$ & Diffuse & - & - & - & Yes & - \\
\hline 4 & Minor & 79 & $15 \cdot 5$ & No & Yes & 140 \\
\hline 5 & Minor & 60 & $6 \cdot 5$ & No & Yes & 99 \\
\hline 6 & Normal & 55 & $13 \cdot 0$ & Yes & Yes & 186 \\
\hline 7 & Normal & 56 & $10 \cdot 5$ & No & Yes & 98 \\
\hline 8 & Normal & 56 & $10 \cdot 6$ & Yes & No & 134 \\
\hline 9 & Moderate & 42 & 9.5 & Yes & Yes & 140 \\
\hline 10 & Normal & 51 & $7 \cdot 0$ & Yes & Yes & 195 \\
\hline 11 & Normal & 44 & $11 \cdot 1$ & Yes & Yes & 103 \\
\hline 12 & Normal & 69 & $12 \cdot 0$ & No. & No & 141 \\
\hline 13 & Normal & 54 & $10 \cdot 0$ & No & No & 125 \\
\hline 14 & Normal & 54 & 14.5 & Yes & No & 97 \\
\hline
\end{tabular}

${ }^{\star}$ Data missing as patient is reviewed abroad.
TABLE II-Perception of quality of life, employment, and number of family dependants in 14 surviving patients

\begin{tabular}{lccc}
\hline Case No & $\begin{array}{c}\text { Quality of } \\
\text { life }^{\star}\end{array}$ & Employment & $\begin{array}{c}\text { Family dependants } \\
\text { (spouse and children) }\end{array}$ \\
\hline 1 & 1 & Full time & 3 \\
2 & 1 & Full time & 3 \\
3 & 1 & Full time & 3 \\
4 & 1 & Full time & 3 \\
5 & 2 & Full time & 3 \\
6 & 2 & Retired (1991) & 4 \\
7 & 1 & Full time & 5 \\
8 & 1 & Unemployed & 2 \\
9 & 3 & Unemployed & 3 \\
10 & 1 & Part time & 2 \\
11 & 1 & Full time & 4 \\
12 & 1 & Full time & 2 \\
13 & 1 & Full time & 5 \\
14 & 1 & Full time & 3 \\
\hline
\end{tabular}

* $1=$ Unrestricted; $2=$ mildly restricted; $3=$ moderately restricted; $4=$ severely restricted in daily activities.

discharge lived more than 5 years and 14 of these were alive at a mean (range) of 69 (61-83) months after surgery. This reflects a $53.8 \%$ long term survival for those who received a suitable donor organ and a $70 \%$ actual survival at almost 6 years' mean follow up in those discharged from hospital after surgery (fig 2).

\section{Discussion}

Though it seems reasonable to assume that the ideal candidate for cardiac transplantation is one who has irreversible cardiac failure and severe symptomatic restrictions despite all treatment but with no requirement for intravenous or interventional support and no secondary end organ damage, in practice many of the patients referred for consideration of transplantation require active support in hospital and have been referred in some cases because they have developed secondary end organ damage. The mortality of these patients approaches $100 \%$ in the short term.

Our long term follow up study of patients who underwent urgent cardiac transplantation shows that many such patients can do excellently, both in terms of survival and quality of life. Previous reports had suggested that such subgroups of patients did well in terms of survival in the short and medium term after transplantation, ${ }^{34}$ assuming that the development of renal failure requiring dialysis had not occurred before receiving a suitable organ, ${ }^{3}$ and quality of life has been shown to increase in absolute terms after such a procedure. ${ }^{5}$ Of the 14 surviving patients in this report, all had required support with intravenous infusions of cardiac drugs before transplantation, and, in addition, some had developed secondary renal and hepatic dysfunction; clearly this does not seem to mitigate against a successful outcome to transplantation. Of the original cohort of 33 patients considered for urgent transplantation, ${ }^{3}$ however, 16 had insertion of an intraaortic balloon pump, but only two of these have survived long term. Although it is difficult to reach firm conclusions from the small number of patients who underwent balloon counterpulsation, those few who have survived in the long term underwent such a procedure while their renal function was still normal or nearly normal and their renal state remained stable until transplantation. Possibly early balloon counterpulsation to offset the expected deterioration in renal function might be a more worthwhile approach to management than later in an attempt to reverse such a deterioration in renal state. If patients with moderate renal and hepatic dysfunction who do not require mechanical intervention before cardiac transplantation survive to hospital discharge their long term outlook is good.

In medicine the issue of economics is becoming evermore prevalent. Cost-benefit for performing cardiac transplantation on such seriously ill patients 
may be looked at more objectively when follow up is longer. Though management in hospital of such patients before transplantation is undoubtedly labour intensive and expensive, many such patients return to normal working life. A further consideration is that many have young families who depend on them. This factor is particularly difficult to price. These issues of survival and quality of life have to be further considered in the light that all such patients in this series who did not receive a suitable donor organ died during their hospital admission. ${ }^{3}$

The long term results of cardiac transplantation are now excellent with five year actuarial survival of almost $70 \%$ when the results of heart transplantation from all institutions are combined. ${ }^{1}$ Much of the improvement in survival has resulted from the now routine use of cyclosporin as an immunosuppressive agent. ${ }^{67}$ Even for severely ill patients a good long term outlook can be expected for most of those undergoing transplantation; the overall survival of $70 \%$ at a mean of 69 months for those discharged from hospital after such an operation compares favourably with the long term results of cardiac transplantation worldwide.

Although no independent assessment of quality of life was performed in these patients, from the objective assessment of exercise capacity and left ventricular function in each case combined with the full time employment of most of the long term surviving patients, the patients' answers to a simple telephone questionnaire were probably accurate and did not reflect any inappropriate bias.

As expected, graft atherosclerosis becomes problematical in long term survivors of cardiac transplantation. ${ }^{8}$ Of the 18 patients who were alive at short term follow up, one has since died of coronary disease; a second patient with coronary disease died of carcinoma of the lung; and four further surviving patients have angiographic evidence of disease. Despite the use of cyclosporin (a nephrotoxic agent) in these patients only two had a plasma creatinine concentration above 175 $\mu \mathrm{mol} / 1$, confirming the findings of Lewis $e t a l^{p}$ that the use of such an agent in the long term was associated with impaired but generally stable aggregate renal function in heart transplant cohorts. von Scheidt $e t$ al $l^{10}$ assessed haemodynamic variables over a 5 year period (mean 26 months) in patients after heart transplantation and confirmed preserved and normal left ventricular contractility in most cases. We have confirmed this finding over a mean of 69 months follow up, with left ventricular ejection fraction ranging from $42 \%$ to $83 \%$.

At the time that the present series of transplant patients were referred for surgery our transplant centre was one of a fairly small number seeking suitable donor organs, not just in Britain but in continental Europe. This allowed for fairly good availability of well selected donor organs thus more easily facilitating an urgent transplant programme. Currently, with strong competition from many units throughout Europe for available organs, many patients similar to those described herein would die waiting for a suitable donor organ. Many more organ donors, however, are available than are being assessed through existing organ procurement efforts. ${ }^{2}$ Donor organs are also possibly lost due to physicians avoiding raising the issue with relatives, although many relatives may find some comfort from knowing that someone else has benefited from their loss. With the chronic shortage, there has been a widening of selection criteria for the acceptance of donor organs and an extension of cardiac allograft ischaemic time ${ }^{11} 12$ in the hope of increasing the available pool of donor organs. While in the short and medium term (up to 3 years) such an approach does not seem to compromise the results of transplantation, 1112 what the long term effects of such an approach will be are unclear.

Non-intra-aortic balloon counterpulsation techniques acting as "bridges to transplantation" were not available to us for clinical purposes in 1985-6, but various recent studies have suggested that well selected patients undergoing such mechanical interventions (ventricular assist device, B ventricular assist device, total artificial heart) have a similar short term outlook to those undergoing "elective" transplantation. ${ }^{1314}$ The expense involved will, however, be justified only by the demonstration of a good long term outlook after such a management approach.

From the findings of this follow up report it seems feasible to combine aggressive treatment of cardiac failure (with its attendant end organ effects) with early cardiac transplantation in severely ill patients. The long term results of transplantation in those requiring intensive support in hospital with infusions of inotropic agents, vasodilators, or both, in addition to more standard treatment seems excellent. If intraaortic balloon counterpulsation is to be used it should perhaps be introduced early in the management of such patients to prevent inexorable deterioration in renal function, rather than late in an effort to reverse such deterioration. This policy will, while potentially reducing the time window for receiving a suitable donor organ, ensure a more optimal clinical state in those who do receive a suitable organ. Patients deteriorating to the point when renal dialysis is considered should be withdrawn from the transplant list. From the results of cardiac transplantation to date many more such patients could expect an excellent long term outlook if referred before developing secondary end organ dysfunction, but more importantly if the supply of available donor organs could be increased.

As of October 1992 all 12 patients living in the United Kingdom were alive and well.

1 Kriett JM, Kaye MP. The registry of the International Society for Heart and Lung Transplantation: eighth official report. $\mathcal{f}$ Heart Lung Transplamt 1991;10:491-8.

2 Evans RW, Orians CE, Ascher NL. The potential supply of donor organs. An assessment of the efficiency of organ procurement efforts in the United States. FAMA 1992;267:239-46.

3 Mulcahy D, Wright C, Mockus L, Yacoub M, Fox KM. Cardiac transplantation in severely ill patients requiring intensive support in hospital. BMF 1988;296:817-9.

4 Hardesty RL, Griffith BP, Trento A, Thompson ME, Ferson PF, Bahnson HT. Mortally ill patients and excellent survival following cardiac transplantation. Ann Thoracic Surg 1986;41:126-9.

5 Bunzel B, Grundbock A, Laczkovics A, Holzinger C, Teufelsbauer H. Quality of life after orthotopic heart transplantation. $\mathcal{f}$ Hear Lung Transplant 1991;10:455-9.

6 Oyer PE, Stinson EB, Jamieson SW, Hunt SA, Perlroth M, Billingham M. Cyclosporine in cardiac transplantation: a $2 \frac{1}{2}$ year follow-up. Transplant Proc 1983;15:2546-52.

7 Dummer JS, Hardy A, Poorsatter A, Ho M. Early infections in kidney, hear and liver transplant recipients on cyclosporine. Transplant 1983;36:259-67.

8 Hosenpud JD, Shipley GD, Wagner CR. Cardiac allograft vasculopathy: current concepts, recent developments, and future directions. $f$ Heart Lung Transplant 1992;11:9-23.

9 Lewis RM, Van Buren CT, Radovancevic B, Frazier OH, Janney RP, Powers $\mathrm{PL}$, et al. Impact of long-term cyclosporine immunosuppressive therapy on PL, et al. Impact of long-term cyclosporine immunosuppressive therapy on kidney transplant recipients. I Heart Lung Transplant 1991;10:63-70.

10 von Scheidt W, Ziegler U, Kemkes BM, Erdmann E. Heart transplantation: hemodynamics over a five-year period. f Heart Lung Transplant 1991;10: hemodyn

1 Menkis AH, Novick RJ, Kostuk WJ, Pflugfelder PW, Powell AM, Thomson $\mathrm{D}$, et al. Successful use of the "unacceptable" heart donor. I Heart Lung Transplant 1991;10:28-32.

12 Pflugfelder PW, Singh NR, McKenzie FN, Menkis AH, Novick RJ, Kostuk WJ. Extending cardiac allograft ischemic time and donor age: effect on survival and long-term cardiac function. $f$ Hean Lung Transplant 1991;10: 394-400.

13 Birovljev S, Radovancevic B, Burnett CM, Vega JD, Bennink G, Lonquist JL. Heart transplantation after mechanical circulatory support: four years' experience. 7 Heart Lung Transplant 1992;11:240-5.

14 Reedy JE, Pennington G, Miller LW, McBride LR, Lohmann DP, Noedel NR. Status 1 heart transplant patients: conventional versus ventricular assist device support. $\mathcal{I}$ Heart Lung Transplant 1992;11:246-52.

(Accepted 23 October 1992) 